\title{
AIO Mitteilungen
}

Kurzfassung des Protokolls AIO 3/90:

A randomized phase- $\prod$-study of intensive dose epirubicin \pm verapamil as induction therapy for metastatic breast cancer patients at high risk

Studienkoordinator: K. Mross

Studienleitung: D. K. Hossfeld

Universitätskrankenhaus Eppendorf, Medizinische Klinik, Abteilung Onkologie/Hämatologie, Martinistr. 52, 2000 Hamburg 20,

Telefon: (040) 468-2959/4854/2960, Telefax: 040/476494

Metastasierte Mammakarzinom-Patienten mit ungünstiger Prognose bedürfen einer intensiven Behandlung. Das Mammakarzinom ist ein chemosensibler Tumor. In einem noch nicht exakt ermittelten Umfang sind P-170-Glykoprotein-haltige Tumorzellen nachweisbar: Ein Indikator für Chemotherapie-resistente Zellen. Anthrazykline bilden die wirksamste Zytostatikaklasse beim Mammakarzinom. Ein Zusammenhang zwischen Dosisintensität und Remissionsrate ist für das Mammakarzinom nachgewiesen.

Das vorliegende Protokoll vergleicht eine intensive Chemotherapie mit einem Anthrazyklin mit der Kombination Anthrazyklin plus resistenzmodulierendes Agens. In vorklinischen und auch in klinischen Studien konnte gezeigt werden, daß Verapamil-resistente Zellen wieder Chemotherapie-sensibel machen kann, indem es den schnellen Efflux des Zytostatikums aus dem Zellinnern blockt.

Die Behandlung sieht die dreimalige Applikation von Epirubicin in drei Chemotherapieblöcken bei zytostatisch nicht vorbehandelten, hormonell vorbehandelten oder nicht vorbehandelten oder adjuvant nur mit CMF vorbehandelten Patientinnen vor. Eine Vorbehandlung mit Anthrazyklinen im Rahmen z. B. einer adjuvanten Therapie ist ein Ausschlußkriterium. Es werden $40 \mathrm{mg} / \mathrm{m} 2$ EPI als i. v. Bolusinjektion an den Tagen 1, 2 und 3 appliziert. Für den Fall, daß EPI mit Verapamil kombiniert wird, ist das Therapieschema bzgl. EPI identisch; zusätzlich wird ab Tag 0 (= ein Tag vor der EPI-Gabe) mit der Gabe von Verapamil begonnen. Dieses Medikament wird alle sechs Stunden mit $120 \mathrm{mg}$ p. o. appliziert (4× $120 \mathrm{mg} / \mathrm{d}$ Verapamil an den Tagen $0,1,2$ und 3 ).

EPI

EPI

EPI

MBCpt high risk

(Randomisierung) 
$\mathrm{MBC}=$ metastatic breast cancer, $\mathrm{pt} \approx$ patient, $\mathrm{EPI}=$ Epirubicin, $\mathrm{VPL}=$ Verapamil

Nach drei Zyklen wird ein Restaging vorgenommen. Prinzipiell ist die Studie an diesem Punkt beendet, da die zu erarbeitenden Punkte in dieser Studie 1. die Toxizität, 2. das Ansprechen auf die Therapie und 3. die Pharmakokinetik und Metabolisierung von EPI und Verapamil sind. Die weitere Therapiestrategie ist den einzelnen Studienteilnehmern freigestellt. Es kann bei Ansprechen auf die Therapie in gleicher Art weiterbehandelt werden, es kann jedoch auch mit anderen Medikamenten weiterbehandelt werden. Selbst eine Therapiepause ist diskutabel. Da die weitere Therapie im Rahmen dieser Studie nicht festgelegt wurde, ist eine Auswertung der Gesamtüberlebenszeit oder - bei den Patienten, die auf die Therapie ansprechen (CR/PR) - der Zeit bis zur Progression nicht vorgesehen, da diese wahrscheinlich stark von dem weiteren therapeutischen Vorgehen abhängen.

Es werden in jedem Arm dieser Studie 14 Patienten benötigt; dies entspricht der klassischen Zahl für Phase-II-Studien. Aufgrund der geringen Fallzahl ist das Aufzeigen eines Unterschieds in beiden Armen bzgl. des Erreichens von Remissionen nicht sehr wahrscheinlich, es sei denn, ein Arm ware extrem effektiver. Im Rahmen dieser Studie wird durch die begleitenden pharmakokinetischen Studien jedoch gezeigt werden können, ob überhaupt Blutspiegel von Verapamil erreichbar sind, die imstande sind, eine Resistenzmodulation zu bewirken. Ferner läßt sich ein Trend - wenn er denn existiert - erkennen, und die Toxizität eines solchen intensivierten Vorgehens wird beurteilbar.

Die Randomisierung erfolgt zentral im Datenzentrum der AIO im Deutschen

Krebsforschungszen-trum in Heidelberg (DKFZ) durch Dr. Edler und Mitarbeiter. Zur Dokumentation sind die Phase-П-Bögen der AIO (Prof. Queisser, Mannheim) zu verwenden. Das Therapieprotokoll kann im AlO-Sekretariat bei Frau Seibel (Hamburg) angefordert werden. Die Behandlung nur mit EPI kann ambulant erfolgen, die Kombination mit Verapamil nur stationär wegen der erhöhten Überwachung (RR-, Puls- und EKG-Kontrolle).

Geschäftsstelle:

Martinistraße 52,

D-2000 Hamburg 20,

Tel. 040/468-2924

Mitteilungen

Eine Weiterentwicklung dieses Studienkonzepts (intensive Chemotherapie mit Resistenzmodulation) ist notwendig und bereits in Planung. Eine weitere Verbesserung ist durch den Einsatz von Wachstumsfaktoren (G-CSF, GM-CSF, IL-3) möglich; die Intensität der Dosis kann bei gleichblei-bender oder sogar reduzierter Toxizität gesteigert werden. Der Ersatz des bezgl. der Toxizität (Herz) kritischen Verapamil duch andere resistenzmodulierende Substanzen (z. B. RVerapamil) wird die Verträglichkeit verbessern. Die Messung des P-Glykoproteins immunhistologisch, zytolo-gisch und mit in-situ-Hybridisierung auf der Ebene der Einzelzelle, mit PCR-Technik und Messung der MDR1 RNA mittels RNA-slot-blot-Technik, oder PGlykoprotein-Nachweis mit Western-blot, werden in künftige Protokolle implementiert werden. Für Patienten mit einem ausgezeichne-ten Ansprechen (schnelles Erreichen einer CR oder einer sehr guten PR) ist auch eine autologe KMTzu diskutieren. 
Geschäftsstelle: Martinistraße 52, D-2000 Hamburg 20, Tel. 040/468-2924

Ergebnisprotokoll der AlO-Vorstandssitzung am 26.06.1990 in Hamburg

Der Vorstand beschließt:

1 Zur Berufspolitik: Der Vorstand der AIO unterstützt die berufspolitischen Forderungen H.-J.

Illigers und U. R. Kleebergs und beschließt dementsprechend die folgenden Maßnah-men:

In einem Rundschreiben werden alle Mitglieder der AIO um Eintritt in den BDI gebeten.

An Veranstaltungen des Berufsverbands Deutscher Internisten und der Deutschen Gesell-schaft

für Innere Medizin sollen AlO-Symposien angekoppelt werden.

Der Präsident der Deutschen Krebsgesellschaft, G. Nagel, wird gebeten, eine Sitzung des

Berufspolitischen Ausschusses und der Vorstände der Arbeitsgemeinschaften anzuberaumen.

Die Vorstände der

Deutschen Gesellschaft für Innere Medizin (Kochsiek),

Deutschen Gesellschaft für Hämatologie und Onkologie (Heimpel),

Deutschen Röntgengesellschaft (Sack) und

Deutschen Krebsgesellschaft (Nagel)

werden gebeten, dem Vorstand der Bundesärztekammer vorzuschlagen, H.-J. Illiger in die

Kommission für Weiterbildung zu berufen.

2. Zu Phase-III-Studien der AIO:

Eingereichte Protokolle werden von mindestens drei Mitgliedern des AIO-PRC (benannt von H.J. Schmoll) beurteilt.

Bei multidisziplinären Studien werden die Protokolle außerdem vom einem oder mehreren

Gutachtern der entsprechenden Arbeitsgemeinschaften beurteilt.

Nach Eingang der Gutachten und eventueller Modifizierung des Protokolls wird in der nächstfolgenden Vorstandssitzung über die Annahme als AlO-Studie entschieden. - Von jedem akzeptierten AlO-Prokoll wird eine Kurzfassung (ca. eine DIN-A4-Seite) an alle AIO-Mitglieder versandt sowie in der ONKOLOGIE veröffentlicht. Außerdem werden Aufstellungen aller laufenden AlO-Studien (Titel und Studienleiter) regelmäßig in ONKOLOGIE und in den MITTEILUNGEN der Deutschen Krebsgesellschaft publiziert. Richtlinien für Phase-III-Studien müssen erarbeitet und eine Qualitätskontrolle muß durchge-führt werden.

Als AlO-Studien werden akzeptiert: AIO 3/89: Postoperative adjuvante Therapie des

Rektumkarzinoms der Stadien Dukes B und

$\mathrm{C}$ mit HDCF + 5-FU mit oder ohne Bestrahlung vs. operierte Kontrollgruppe.

Studienleitung: A. Knuth, Mainz. AIO 4/89: HDCF + 5-FU vs. NDCF + 5-FU bei

fortgeschrittenen kolorektalen Karzinomen.

Studienleitung: A. Knuth, Mainz. AIO 2/90: Therapie der CLL im Frühstadium: Wirksamkeit von rekombinantem IFN-alpha

bei Patienten mit hohem Risiko für eine Krankheitsprogression. Studienleitung:

B. Emmerich, München; E. Thiel, Berlin. AIO 3/90: Intensive dose epirubicine \pm verapamil as induction therapy for metastatic breast

cancer patients at high risk. Studienleitung: D. K. Hossfeld, K. Mross, Hamburg. Die

Arbeitsgruppe «Gastrointestinaltumoren» wird gebeten, ein Studienprotokoll für die Prüfung von

Levamisol zu erstellen. Vorgeschlagen wird eine zwei- oder dreiarmige Studie: 5-FU+

Levamisol vs. 5-FU + Leucovorin (vs. 5-FU Monotherapie). 
Um die Effizienz der organbezogenen Arbeitsgruppen zu erhöhen, wird ein Schema als Leitlinie für die Strukturierung der Arbeitsgruppen erarbeitet. Dieses Schema wird den Leitern der Arbeitsgruppen übermittelt, ohne daß es allerdings verbindlich wird. 1st ein pharmazeutisches Unternehmen in eine AIO-Phase-IП-Studie involviert, wird pro Patient ein Fallgeldbetrag von DM 1000,- als Richtlinie festgelegt. Von diesem Betrag gehen DM 250,- an die AIO und DM 750,- an den Prüfarzt. 1st kein Unternehmen beteiligt, gibt es keine Fallgelder.

Mitteilungen

3. Zur Aufnahme neuer Mitglieder:

Als ordentliche Mitglieder werden aufgenommen: C. Boewer (Berlin) und R.-M. Zippel (Nürnberg). - Als außerordentliche Mitglieder werden aufgenommen: A. Harstrick (Hannover), M. Schmid (Graz) und T. A. Walter (Hamburg).

Die Geschäftsordnung der AIO wird ergänzt im Hinblick auf Referenzen bei Bewerbungen um außerordentliche Mitgliedschaft.

4. Zur Anfrage von U. Gunzer anläßlich der AIO-Mitgliederversammlung im März d.J. (s. Protokoll der MV, TOP 10):

Da der Vorstand der AIO nicht von den Krankenkassen verlangen kann, daß diese experimentelle Therapien finanzieren, sollen die betreffenden Patienten nach Möglichkeit im Rahmen von Studien behandelt werden. 1st dies nicht möglich, sind direkte Verhandlungen mit der zuständigen Kasse zu führen.

5. Zur Leitung der Arbeitsgruppe «Urogenitaltumoren»:

H.-J. Schmoll bleibt alleiniger Leiter der Arbeitsgruppe «Urogenitaltumoren».

Hamburg, den 18.07.1990

Bericht über das Symposium «lnterferone - Biologische Grundlagen und klinische Anwendung» Hamburg, 26.-27. Januar 1990

Die Veranstaltung unter Leitung von Prof. Dr. N. Niederle und Prof. Dr. D. K. Hossfeld diente zunächst einer möglichst umfassenden Bestandsaufnahme der bisher mit Interferonen erzielten präklinischen und klinischen Befunde in der Hämatologie und Onkologie.

Über die einzelnen Aspekte, wie Physiologie und Pathophysiologie des Interferonsystems, Proteinchemie der Interferone, biologische Wirkung, Rezeptoren, Second-Messenger, Antikörper, Toxizität, präklinische Befunde und klinische Ergebnisse bei hämatologischen Systemerkrankungen und soliden Tumoren sowie die Kombinationsmöglichkeiten mit anderen zytoreduktiven Therapiemaßnahmen, wurde durch eingeladene kompetente Redner umfassend berichtet. Aus dieser Darstellung der bisherigen Befunde und Behandlungsmöglichkeiten ergaben sich in der an jeden Vortrag direkt anschließenden Diskussion Ausblicke auf zukunftsweisende pathogenetische Aspekte und neue diagnostische sowie therapeutische Ansätze. In diesem Zusammenhang ist an die noch sehr präliminären Ergebnisse zum Wirkmechanismus und zum Proteindesign sowie an die Erstellung aussagefähiger prädiktiver Testsysteme für eine Optimierung der klinischen Anwen-dung zu erinnern. Darüber hinaus erscheint es wichtig, prospektive Studien zur Interferon-Monotherapie und - wo angezeigt - zur Kombination von Interferonen mit anderen Zytokinen, aber auch Zytostatika, zu initiieren und sauber zu evaluieren, so daß in absehbarer Zeit der Wert der Interferone im Behandlungskonzept verschiedener maligner Erkrankungen sicherer definiert werden kann.

Workshop: «Modifícation of Drug Resistance* Aachen, 23.06.1990

Unmittelbar nach dem Einsatz des ersten Zytostatikums (Mustargen) in der Klinik stellte sich das Problem der primären und der erworbenen Resistenz. Langjährige Vorarbeiten über Resistenz gegenüber alkylierenden Zytostatika und Antimetaboliten erzielten eindrucksvolle Ergebnisse 
bezüglich einer Wirkungsverstärkung. Aber erst Tsuruo hat mit dem aufsehenerregenden Begriff Resistenzüberwindung («reversal of resistance») die Aufmerksamkeit der klinisch tätigen

Onkolo-gen verstärkt auf dieses Arbeitsgebiet gelenkt.

Geschäftsstelle:

Martinistraße 52,

D-2000 Hamburg 20,

Tel. 040/468-2924

Mitteilungen

Ziel des Workshops war zunächst eine kritische Sichtung und Wertung der bisher erreichten Ergebnisse zur Modifikation der Resistenz gegenüber Zytostatika. Alle Vortragenden unternahraen den Versuch, von den molekularpharmakologischen Grundlagen über In-vitro-

Testergebnisse und Tierversuche bis hin zur Phase-I- und -П-Testung inklusive Pharmakokinetik die jeweiligen Ergebnisse zusammenhängend darzustellen.

M. Dietel (Kiel) gab eine mit hervorragendem Bildmaterial ausgestattete Einführung über morphologische Veränderungen auf lichtmikroskopischer und ultrastruktureller Ebene bei Tumorzellen mit dem sogenannten «MDR-Phänotyp», aber auch bei normalen menschlichen Geweben. W. T. Beck (Memphis, TN, USA) zeigte, daß neben der bekannten Form der «multidrug resistance» atypische Formen auftreten, die mit Änderungen am Topoisomerase-Komplex zusam-menhängen.

B. Hill (London, England) berichtete über ihre überraschenden Ergebnisse zur Induktion von Resistenz gegenüber Zytostatika durch fraktionierte Vorbestrahlung. Die breite Rolle von Glutathion in der Detoxifikation von chemischen Noxen inklusive einer zytostatischen Behandlung und ihrer Nutzung für die Modifikation der Resistenz gegenüber Alkylantien, wurde von R. F. Ozols (Philadelphia, PA, USA) geschildert. S. B. Kaye (Glasgow, Schottland) nahm zur Möglichkeit der Modulation des «MDR-Phänotyps» durch Kalzium-Antagonisten Stellung und verwies auf das weniger kardiotoxische R-Enantiomer des Racemats Verapamil.

Neben der direkten Einwirkung auf das P-Glykoprotein kann Cyclosporin A nach K. J. Scanlon (Duarte, CA, USA) auch die Onkogenexpression in Cisplatin-resistenten Zellen verändern. Die vorgetragenen Untersuchungen belegen eindrucksvoll, daß die resistenzmodifizierenden Substan-zen, die sich bekanntlich aus zahlreichen Wirkstoffgruppen rekrutieren, eine Vielzahl von Wirkun-gen auf Tumorzellen im Zusammenhang mit der Applikation von Zytostatika ausüben können. Die umfangreichen Erfahrungen mit der biochemischen Modulation von 5Fluorouracil wurden von R. Herrmann (Berlin) kritisch gewürdigt. In einem Posterbeitrag stellte U. Fabry (Aachen) eine Wirkungsverstärkung sowohl bei sensiblen als auch 5-Fluorouracilresistenten Zellen fest. H. J. Schmoll (Hannover) fand klinisch keine Wirksamkeitsverstärkung durch Dipyridamol, das nach seinen Ausführungen unter In-vitro-Bedingungen ein enormes Spektrum an Möglichkeiten der Wirkungsverstärkung aufweist.

M. Scheulen (Essen) referierte über die neueren Entwicklungen bei Antrazyklinen und Platinkom-plexen. Bestimmte strukturelle Modifikationen führen zur Abwesenheit einer Kreuzresistenz mit den Ausgangssubstanzen. Unklar bleibt jedoch der klinische Nutzen dieser neuen Substanzen, die ein deutlich verändertes Toxizitätsspektrum aufweisen. Abschheßend erhob sich die Frage, wie sich das Resistenzproblem für die mit vielen Erwartungen und Hoffnungen verknüpften sogenannten «BRM-Substanzen» darstellt. O. Kloke (Essen) gab für die Interferone eindrucksvolle Beispiele, wie neoplastische Zellen auch gegenüber diesem völlig neuartigen Wirkprinzip resistent werden können. 
Die Posterbeiträge ergaben einen ausgezeichneten Überblick über den Stand der Resistenzforschung, insbesondere in der BRD; aber auch aus den benachbarten Benelux-Ländern, England und den USA wurden Beiträge eingereicht. Molekularbiologische, zytogenetische, zytophotometrische Methoden sowie In-vitro-Testungen und klinische Studien wurden im Zusammenhang mit dem Resistenzproblem dargestellt. Es kann erwartet werden, daß aus diesem neuen Arbeitsgebiet der Resistenzmodifikation wesentliche Impulse ausgehen, um das derzeitige Plateau bei der Behandlung von fortgeschrittenen Tumorerkrankungen mit konventionellen Zytostatika zu über-winden. Gleichzeitig wurde klar, daß auch für neuartige Wirkstoffe die Problematik der Resistenz aufgrund der enormen Plastizität von Tumorzellen ein Kernproblem werden wird.

Geschäftsstelle: Martinistraße 52, D-2000 Hamburg 20, Tel. 040/468-2924

Farmitalia-Carlo-Erba-Preis 1990

Am 19.10.1990 wurden anläßlich des Symposiums «Bronchialkarzinome - Diagnostische und therapeutische Aspekte 1990» die Farmitalia-Carlo-Erba-Preise 1990 verliehen. Von 5 eingereichten Publikationen hatte die Jury 2 als preiswürdig befunden. Bei der Urteilsbil-dung spielten der innovative Ansatz, die Qualität der Methodik und die klinische Bedeutung eine wichtige Rolle.

Die Preisträger sind:

«Tumorhemmende Potenz und Wirkmechanismus von Antigestagenen in experimentellen Mammakarzinomen»

H. Michna und M. R. Schneider (Forschungslaboratorium der Schering AG, Berlin)

Die Arbeit ist die Zusammenfassung von drei Publikationen, die sich mit dem antitumoralen Effekt von zwei Antigestagenen (Mifepriston und Onapriston) befassen. Dieser Arbeit liegen umfangreiche tierexperimentelle Untersuchungen an hormonabhängigen Mammakarzinomen der Maus und der Ratte einschließlich morphologischer und Endokrin-pharmakologischer Untersuchungen zugrunde. Beide Substanzen zeigten im Experiment eine ausgeprägte antitumorale Wirkung, deren Effekte denen von Tamoxifen oder hochdosierten Gestagenen überlegen waren. Von großem Interesse sind die Endokrin-pharmakologischen Untersuchungen, die eindeutig darauf hinweisen, daß der antitumorale Effekt nicht auf eine Hormondeprivation zurückzuführen ist. Beide Substanzen zeigen im Gegenteil eine Aktivierung der Ovarialfunktion mit Anstieg der entsprechenden Hormonspiegel. Der tumorhemmende Effekt wird auf noch unbekanntem Wege über Progesteronrezeptoren vermittelt und führt nicht zu Tumornekrosen, sondern zu einer Differenzierung der Karzinome.

Die Jury würdigte den innovativen Ansatz der experimentellen Arbeiten, deren Ergebnisse durch umfangreiche und gut dokumentierte Untersuchungen belegt sind. Die Arbeiten enthalten die Grundlagen für eine neue und wirkungsvolle Therapie für das hormonabhängige Mammakarzinom.

Mitteilungen

Kongresse und Fortbildungsveranstaltungen 1991:

18.1.-19.1. «Cytokines as Modulators of Cytotoxic Drugs in Experimental and Clinical Hematology and Oncology», Symposium der AIO, Berlin 1.2.-2.2. «Die Therapie des chronisch Krebskranken», Interdisziplinäres Symposium, Essen 
24.2-26.2 «Acute Leukemias - Pharmacokinetics and Management of Relapsed and Refractory Disease», Symposium der Deutschen AML-Arbeitsgruppe, des M. D. Anderson Cancer Center, der DGHO und AIO, Münster

11.3.-13.3. 4. Ärzte-Fortbildungskurs in klinischer Onkologie für Vorgerückte, SAKK, AIO, St. Gallen

20.3.-21.3. «Ernährung und Tumorerkrankungen», Vorsymposium anläßlich der 10. gemeinsamen Jahrestagung der DAKE/AKE, Göttingen

11.4.-13.4. Symposium zum 1Ojährigen Bestehen des Farmitalia Carlo-Erba-Preises, (nur für eingeladene Teilnehmer), Freiburg

28.11.-29.11. Symposium der Phase-I/II-Studiengruppe der AIO (Titel steht noch nicht fest), Dresden

1992

25.6.-27.6.

2nd Frankfurt International Cytokine-Symposium, AIO, DK, Tumorzen-trum Frankfurt Geschäftsstelle:

Martinistraße 52,

D-2000 Hamburg 20,

Tel. 040/468-2924 